\title{
Slow Conduction through an Arc of Block: A Basis for Arrhythmia Formation Post-Myocardial Infarction
}

Short title - Reflection and PVB Formation Post MI

\section{Eugene Patterson, PhD, Benjamin J. Scherlag, PhD, Edward J. Berbari, PhD, and Ralph Lazzara, MD}

Heart Rhythm Institute, The University of Oklahoma Health Sciences Center and OU Medical Center, Oklahoma City, OK

Eugene Patterson, PhD, Department of Integrative Physiology and Pharmacology, Liberty University College of Osteopathic Medicine, Lynchburg, VA

This is the author's manuscript of the article published in final edited form as:

Patterson, E., Scherlag, B. J., Berbari, E. J. and Lazzara, R. (2017), Slow conduction through an arc of block: A basis for arrhythmia formation post-myocardial infarction. J Cardiovasc Electrophysiol. Accepted Author Manuscript. http://dx.doi.org/10.1111/jce.13300 
Benjamin J. Scherlag, PhD, Heart Rhythm Institute, Department of Medicine, The Heart Rhythm Institute at the University of Oklahoma Health Sciences Center and OU Medical Center, Oklahoma City, OK.

Edward J. Berbari, PhD, Biomedical Engineering Program, Indiana University Purdue University, Indianapolis, IN.

Ralph Lazzara, MD, Heart Rhythm Institute, Department of Medicine, The University of Oklahoma Health Sciences Center and OU Medical Center, Oklahoma City, OK.

\author{
Address for Correspondence: $\quad$ Eugene Patterson, PhD \\ Liberty University \\ College of Osteopathic Medicine \\ 306 Liberty View Lane \\ Lynchburg, VA 24502 \\ $(434)-592-6987 \quad$ phone \\ espatterson@liberty.edu
}

Supported by a research grant from the American Heart Association, Heartland Affiliate

This article is protected by copyright. All rights reserved. 


\section{Disclosures: None}

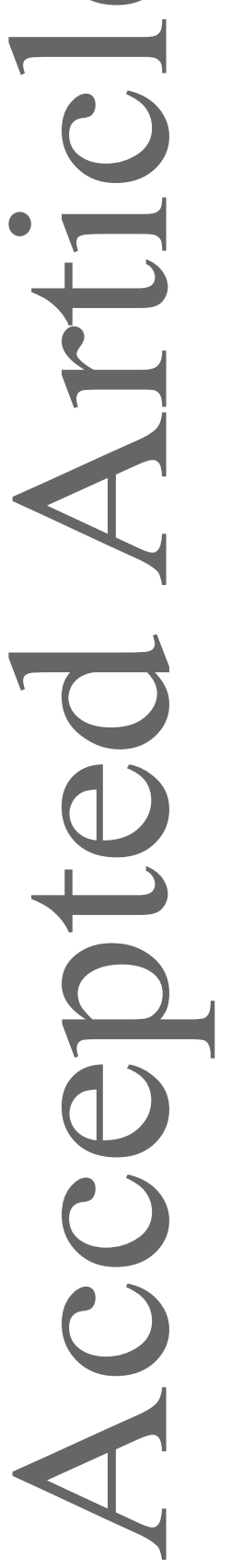

This article is protected by copyright. All rights reserved. 


\section{Abstract}

Introduction - The electrophysiologic basis for characteristic rate-dependent, constant-latecoupled (390+54 msec) premature ventricular beats (PVBs) present 4-5 days following coronary artery occlusion were examined in 108 anesthetized dogs.

Methods and Results - Fractionated/double potentials were observed in injured zone bipolar and composite electrograms at prolonged sinus cycle lengths $(1296 \pm 396 \mathrm{msec})$. At shorter cycle lengths, conduction of the delayed potential decremented, separating from the initial electrogram by a progressively prolonged isoelectric interval. With sufficient delay of the second potential following an isoelectric interval, a PVB was initiated. Both metastable and stable constant-coupled PVBs were associated with Wenckebach-like patterns of delayed activation following an isoelectric interval. Signal-averaging from the infarct border confirmed the presence of an isoelectric interval preceding the PVBs $(N=15)$. Pacing from the site of double potential formation accurately reproduced the surface ECG morphology $(\mathrm{N}=15)$ of spontaneous PVBs. Closely-spaced epicardial mapping demonstrated delayed activation across an isoelectric interval representing "an arc of conduction block". Ratedependent very slow antegrade conduction through a zone of apparent conduction block $(\mathrm{N}=8)$ produced decremental activation delays until the delay was sufficient to excite epicardium distal to the original "arc of conduction block", resulting in PVB formation.

Conclusion - The present experiments demonstrate double potential formation and ratedependent constant-coupled late PVB formation in infarcted dog hearts. Electrode recordings demonstrate a prolonged isoelectric period preceding PVB formation consistent with very slow conduction ( $<70 \mathrm{~mm} / \mathrm{sec}$ ) across a line of apparent conduction block and may represent a new mechanism of PVB formation following myocardial infarction.

This article is protected by copyright. All rights reserved. 
Keywords: myocardial reentry, myocardial infarction, arrhythmia formation, ventricular arrhythmia, ventricular premature beats

This article is protected by copyright. All rights reserved. 


\section{Introduction}

Arrhythmia observed following coronary artery ligation in the dog falls into a number of distinct temporal phases having a variety of electrophysiologic mechanisms ${ }^{1}$. Although rapid and sustained reentrant ventricular arrhythmias can be induced by programmed electrical stimulation, 4-5 days following left anterior descending coronary artery ligation ${ }^{2,3}$, the spontaneous ventricular arrhythmias observed following myocardial infarction have largely been attributed to a slow resolution of residual abnormal automaticity or delayed afterdepolarization formation in endocardial Purkinje tissues surviving myocardial infarction. This form of arrhythmia begins within a few hours following coronary artery ligation ${ }^{4,5}$. In the present studies, we describe a rate-dependent arrhythmia consisting of constant-latecoupled PVBs having a normal axis and left bundle branch block morphology, originating from ischemically-injured (IZ) anterior left ventricular epicardium. The metastable or stable arrhythmias (bigeminal, trigeminal, quadrigeminal, etc., single PVBs) are manifest as a function of the atrial heart rate. In the present studies, we employed a variety of different electrical recording techniques (bipolar, bipolar-composite, signal-averaging, and unipolar epicardial mapping) to investigate the electrophysiologic mechanism giving rise to these arrhythmias.

This article is protected by copyright. All rights reserved. 


\section{Methods}

Surgical preparation - Male dogs were anesthetized with intravenous sodium pentobarbital (30 mg/kg). A cuffed endotracheal tube was inserted and the animals were ventilated with room air using a Harvard respirator. Using aseptic technique, a left thoracotomy was performed in the 5th intercostal space and the heart was suspended in a pericardial cradle. The left anterior descending coronary artery was dissected from surrounding tissue at the tip of left atrial appendage (approximately $1-1.5 \mathrm{~cm}$ from its origin) and the artery ligated in two stages as described by Harris ${ }^{4}$. The chest was closed in layers. Ampicillin $(10 \mathrm{mg} / \mathrm{kg})$ and nalbuphine $(0.2 \mathrm{mg} / \mathrm{kg})$ were administered IM, and the animals were allowed to recover from anesthesia. All animal studies were approved by the Institutional Animal Care and Use Committees of the University of Oklahoma Health Sciences Center and Oklahoma City Veterans Administration Medical Center. All experiments conformed to the Guiding Principles of the American Physiologic Society.

Electrophysiologic Studies - On the $4^{\text {th }}$ or $5^{\text {th }}$ day following coronary artery ligation, the dogs were again anesthetized with intravenous sodium pentobarbital (30 mg/kg). A cuffed endotracheal tube was inserted and the animals were ventilated with room air using a Harvard respirator. A 12-lead surface ECG was obtained. An electrode catheter was inserted through a carotid artery to record His bundle activation $(30-1000 \mathrm{~Hz})$. Body temperature was maintained at $37 \pm 1^{\circ}$ by a heating blanket. Arterial blood pressure was measured from a femoral artery and continuously monitored. Drug administration was performed through a femoral vein. All recordings were obtained using (1) an Electronics for Medicine VR-16 and Gould electrostatic recorder or (2) a Bard computer-based recording system. The right cervical vagus nerve was exposed and $0.1 \mathrm{~mm}$ diameter silver wires were inserted for nerve stimulation (0.01 msec duration, $20 \mathrm{~Hz}, 1-20 \mathrm{~V})$ using a Grass model S-88

This article is protected by copyright. All rights reserved. 
stimulator. Plunge bipolar stainless steel wires $(0.13 \mathrm{~mm}$ diameter $)$ coated with Teflon ${ }^{\circledR}$ were inserted into right ventricular epicardium within the right ventricular outflow tract to allow provocative right ventricular pacing to induce sustained reentrant ventricular tachycardia as described previously 6 .

A left thoracotomy was performed in the 5th intercostal space and the heart was suspended in a pericardial cradle. Atrial pacing was performed from the right or left atrial appendage (4.0 msec duration stimuli at twice diastolic threshold voltage). Electrical recordings were serially obtained from ischemically-injured myocardium using (1) epicardial bipolar composite electrodes, (2) a handheld epicardial probe (two $1 \mathrm{~mm}$ diameter silver electrodes located $2 \mathrm{~mm}$ apart in epoxy), (3) $1 \mathrm{~mm}$ diameter silver electrodes located approximately $5 \mathrm{~cm}$ apart in an $\mathrm{X}$ (epicardial), $\mathrm{Y}$ (epicardial), and $\mathrm{Z}$ (transmural) (orthogonal) orientation grid, (4) a 124 unipolar electrode array with $4 \mathrm{~mm}$ interelectrode spacing, and (5) an $11 \times 11$ epicardial electrode grid $(0.1 \mathrm{~mm}$ silver wire, $1 \mathrm{~mm}$ electrode separation in epoxy). Composite electrograms, orthogonal, and local bipolar electrograms were filtered at 30 and $1000 \mathrm{~Hz}$. Unipolar electrograms for cardiac mapping were filtered at 0.1 and 1000 $\mathrm{Hz}$, undergoing $\mathrm{AD}$ conversion at $2 \mathrm{KHz}$ with 12 bit accuracy. Timing of unipolar electrograms for isochronal mapping was performed using the maximum negative differential and verified manually. Signal-averaging from $X, Y$, and $Z$ orthogonal leads from the IZ borders was compared during sinus rhythm and during rate-dependent rhythm formation (atrial pacing). During arrhythmia formation, the PVB was utilized as the template for signal averaging. A total of 200 intervals preceding the template matched PVB were averaged using Predictor ${ }^{\circledR}$ software (Corazonix).

Statistical Analysis - Data are expressed as the mean \pm the standard deviation. Differences between groups were determined by an analysis of variance for paired or This article is protected by copyright. All rights reserved. 
unpaired data as appropriate followed by Student-Newman-Keuls analysis (Graphpad Instat version 2.1). Statistical significance was assumed at a $p$ value $\leq 0.05$.

This article is protected by copyright. All rights reserved. 


\section{Results}

Patterns of Arrhythmia Formation - The present studies were performed in 108 dogs exhibiting constant-coupled, rate-related ventricular ectopic beat formation with during sinus rhythm $(\mathrm{N}=45)$ or during atrial pacing $(\mathrm{N}=63)$ (Figure 1). Each dog demonstrated transmural myocardial infarction $(Q$ wave formation on at least three consecutive anterior ECG leads V2 - V6). The arrhythmia was often present during sinus rhythm prior to thoracotomy and was also observed after thoracotomy. The constant-coupled, single, premature ventricular beats uniformly demonstrated a normal ECG axis and a left bundle branch block ECG morphology. The slowest atrial rate producing constant-coupled, latecoupled PVBs was $109 \pm 34 \mathrm{bpm}$. The pattern and coupling interval for ventricular ectopic beat formation were reproducible over a narrow range of atrially-paced cycle lengths. The coupling intervals of the ventricular ectopic beats were typically prolonged $(X \pm S D, 390 \pm 54$ msec) and exceeded the duration of the QT interval by $60-200$ msec. Fusion and concealed fusion beats were commonly observed. The pattern of PVB formation was reproducible and rate-dependent, with rate-suppression of PVB formation at shorter paced atrial cycle lengths and at longer atrial cycle lengths produced by vagus nerve stimulation (Figures 1B and 2). Note in Figure 1B the absence of PVB formation at atrial cycle lengths < 360 msec and $>450$ msec with bigeminy, trigeminy, and quadrigeminy observed at intermediate cycle length (Figure 1A and 1B). Although the intervals varied considerable for different individual experimental preparations, the results were consistent in vivo using different localized recording techniques.

Delayed $(>70 \mathrm{msec})$ fractionated potentials or double potentials $(X \pm S D=148 \pm 40$ msec vs. $70 \pm 9 \mathrm{msec}$, normal myocardium) were observed in composite bipolar electrograms recorded from ischemically-injured (IZ) epicardium in each animal at prolonged

This article is protected by copyright. All rights reserved. 
atrial cycle lengths produced by right vagus nerve stimulation $(X \pm S D=1296 \pm 396 \mathrm{msec}, \mathrm{N}$ $=103)$. At shorter sinus or atrially-paced ventricular cycle lengths, splitting and decremental conduction of the terminal portion of the IZ electrograms was observed (Figure 2). The initial and terminal fragments of the IZ electrogram were separated by a prolonged isoelectric interval. If activation of the terminal fragment of the IZ electrogram was sufficiently late within diastole (> 60 msec past the QT interval), a PVB was elicited (Figures 2 and 3). Stable patterns of ventricular beat formation (2:1, 3:1, 4:1, 5:1) (Figures 1 and 2) were observed associated with stable Wenckebach-like patterns of delayed activation. Stable patterns of Wenckebach-like delayed IZ epicardial activation following isoelectric intervals, resulting in the stable patterns of ventricular ectopy formation that are shown in Figures 1 and 2.

A lead-II surface ECG, His-bundle electrograms, normal (NZ) and ischemicallyinjured zone (IZ) composite electrograms, and $X, Y$, and $Z$ orthogonal leads for a single experiment are shown in Figure 2. At an atrial cycle length of $760 \mathrm{msec}$ produced by right vagus nerve stimulation (Figure $2 \mathrm{~A}$ and upper half of Figure $2 \mathrm{~B}$ ), moderately delayed activation was observed in the IZ composite and $Z$ orthogonal electrograms, with stable $1: 1$ activation and no ventricular arrhythmia formation. During sinus rhythm (425 msec) (Figure 2C), a stable 2:1 pattern of delayed/markedly delayed activation was observed in IZ composite and $Z$ orthogonal electrograms. The prolonged delay following the $2^{\text {nd }}$ beat $(>$ $200 \mathrm{msec}$ ) isoelectric interval initiated stable, repetitive 3:1 PVB formation (trigeminy). A slightly more prolonged sinus cycle length of $488 \mathrm{msec}$ produced a repetitive decremental Wenckebach-like conduction pattern of a delayed second IZ potential with successive isoelectric intervals of 0,132 , and 200 msec. Only the last potential following an isoelectric interval of 200 msec produced a PVB (Figure 2D).

This article is protected by copyright. All rights reserved. 
Localized Bipolar Recordings - A bipolar electrode with two $1 \mathrm{~mm}$ diameter silver electrodes located $2 \mathrm{~mm}$ apart in epoxy was used to record from IZ ventricular epicardium to localize the site of rate-dependent PVB formation in 59 infarcted canine hearts. A total of 123 sites of double potential formation were recorded (range $=1-4$ sites/heart). Using atrial-pacing or vagus nerve stimulation, PVB formation was observed at 78 sites of double potential formation. Arrhythmia formation was demonstrated at only a single site in 41 hearts, at two sites in 14 hearts, and at three sites in 3 hearts. When multiple sites of double potential formation were present, each site had a different and distinct cycle length producing Wenckebach-like delays and each site of double potential formation produced a ventricular beat with a slightly different ECG morphology. Each PVB form had a left bundle branch block morphology and normal axis. An example of arrhythmia formation associated with a double potential recorded by a closely-spaced bipolar electrode is shown in Figure 3 (A-C). During sinus rhythm (panel A) (355 msec sinus cycle length), stable 2:1 block is observed in the bipolar electrogram at a site of double potential formation (V1, V2). The 2:1 block became stable 1:1 conduction with vagus nerve stimulation-induced prolongation of the sinus cycle length (panel B) (A-A interval $=835 \mathrm{msec})$. At an intermediate sinus cycle length produced by vagus nerve stimulation (panel C) (430 msec), Wenckebach-like conduction delays of the second potential (V2) were observed, and when sufficiently delayed, produced fusion PVBs. Atrial cycle lengths $>600$ msec (Figure 3B) and $<400$ msec (not shown) failed to elicit localized periodic conduction (Wenckebach-like conduction) or PVB formation.

In 8 experiments, epicardial pacing from the site of double potential formation overlying the infarcted myocardium was performed. Pacing from a double potential

This article is protected by copyright. All rights reserved. 
recording site closely reproduced the morphology of the spontaneous beat originating from the same double potential site as verified by a bipolar recording(6 lead ECG) (Figure 3D).

Signal-Averaged Recordings from Infarcted Myocardium - In 15 experiments, X, $\mathrm{Y}$, and $\mathrm{Z}$ orthogonal bipolar electrograms were recorded from the lateral borders and from endocardium to epicardium in the center of the anterior infarct during normal sinus rhythm (Figure 3E). The PVB was utilized as the template during stable ventricular arrhythmia formation produced by atrial pacing. In Figure 3E, note the presence of delayed lowervoltage activity seen in the $\mathrm{X}, \mathrm{Y}$, and $\mathrm{Z}$ leads during sinus rhythm and the absence of that electrical activity in the atrially-paced beat preceding a PVB with an intervening isoelectric interval of $225 \mathrm{msec}$. This period of electrical diastole was consistent with the electrical diastole present in both bipolar and composite $I Z$ electrograms recorded in the same experiment.

Epicardial Mapping of IZ Activation - Epicardial mapping was initially performed using a unipolar electrode array using unipolar electrodes with a $4 \mathrm{~mm}$ interelectrode distance $(\mathrm{N}=126)$. The delayed $\left(2^{\text {nd }}\right)$ potential of a double potential as well as Wenckebachlike conduction delays of the $2^{\text {nd }}$ potential during atrial pacing were observed only at a single unipolar recording site in each experiment. In each experiment, variable conduction delay and earliest electrical activity for the PVB were observed only at a single recording site only $4 \mathrm{~mm}$ away from multiple sites demonstrating 1:1 activation, implying very slow conduction and block within a $4 \mathrm{~mm}$ or smaller distance. Higher density unipolar electrode resolution epicardial mapping experiments were then performed.

An $11 \times 11 \mathrm{~mm}$ electrode array (unipolar electrodes embedded $1 \mathrm{~mm}$ apart in epoxy) was used to record from IZ epicardium and make activation maps during sinus rhythm, during sinus bradycardia produced by vagus nerve stimulation, and during ventricular

This article is protected by copyright. All rights reserved. 
arrhythmia formation $(N=13)$. Delayed activation $(X \pm S D=128 \pm 14 \mathrm{msec})$ was observed at a heart rate of $120 \pm 15 \mathrm{bpm}$ during vagus nerve stimulation $(\mathrm{N}=4)$ or sinus rhythm $(\mathrm{N}=9)$.

In one example (Figure 4), a double potential was observed during sinus rhythm (upper left hand panel) (134 bpm). The latest site of epicardial activation was $124 \mathrm{msec}$. Slowing the sinus heart rate with vagus nerve stimulation (upper right hand panel) (80 bpm) reduces the maximal epicardial activation delay of the double potential to $102 \mathrm{msec}$. At a paced atrial heart rate of $156 \mathrm{bpm}$, ventricular arrhythmia formation (trigeminy) was observed. A double potential was observed on beat one, with local bipolar electrode and epicardial activation maps (maximal delay of $130 \mathrm{msec}$, respectively, not shown) very similar to those shown for sinus rhythm. The second paced beat demonstrated a truncated (single) electrogram and activation map (Figure 6, lower left hand panel), with a prolonged isoelectric delay (128 to $224 \mathrm{msec}$ ) preceding the second potential of the local bipolar electrogram (Figure 4, lower right hand panel), and initiating a PVB. The isoelectric interval was $96 \mathrm{msec}$ with the earliest activation of the PVB occurring at a site $2 \mathrm{~mm}$ away from the site of latest activation for the preceding sinus beat, across an apparent line of conduction block for the sinus beat and the premature ventricular beat (Figure 4, lower right hand panel).

In a second experiment presented in Figure 5, ventricular arrhythmia was observed during sinus rhythm (146 bpm) in a pattern of ventricular trigeminy associated with splitting of a double potential recorded from a bipolar electrode on the epicardial surface overlying the anterior infarct. During the first beat of sinus origin (Figure 5, upper left hand panel), a maximal delay of 128 msec was observed. During the second beat of sinus origin (the sinus beat immediately preceding the PVB), eight unipolar epicardial electrode recording sites demonstrated no electrical activity during the pre-PVB sinus beat (Figure 5, upper right hand panel). Subsequent PVB formation originated from a site that was not excited during the

This article is protected by copyright. All rights reserved. 
preceding sinus beat and was $1.0 \mathrm{~mm}$ distant from and $190 \mathrm{msec}$ later than the last site activated during the preceding sinus beat. Despite the prolonged delay, conduction from the site of earliest activation was initially very slow $(<50 \mathrm{~mm} / \mathrm{sec})$ until leaving the zone of previously inexcitable epicardium (Figure 5, lower left hand panel).

In each of 10 examples recorded from eight canine hearts, earliest PVB activation was recorded immediately distal to and adjacent to an arc of apparent conduction block. The site of earliest PVB activation was $1.4 \mathrm{~mm}(\mathrm{~N}=2), 2.0 \mathrm{~mm}(\mathrm{~N}=2), 2.2 \mathrm{~mm}(\mathrm{~N}=1), 2.8$ $\mathrm{mm}(\mathrm{N}=3)$, or $4 \mathrm{~mm}(\mathrm{~N}=2)$ across an arc of apparent conduction block for the preceding beat (Table 1). The site of earliest PVB activation was observed distal to the arc of conduction block, following a failure for delayed activation at the distal site by conduction around an arc of block, and was suggestive of very slow conduction "through" the "arc of conduction block" during a time period lasting $(108 \pm 65 \mathrm{msec})(2.4 \pm 1.2 \mathrm{~mm}$ distance)(Figure 6).

Relationship to Sustained Ventricular Tachycardia - Pacing-induced sustained ventricular tachycardias (173 $\pm 21 \mathrm{msec}$ cycle length) were observed in only 5 of 48 animals tested using right ventricular pacing. IZ composite electrograms in canine hearts demonstrating sustained monomorphic ventricular tachycardia demonstrated less activation delay on the epicardial surface during sinus rhythm $(98 \pm 18 \mathrm{msec})(\mathrm{N}=5)$ than animals demonstrating rate-dependent PVB formation during sinus rhythm or atrial pacing $(133 \pm 36$ msec) $(N=43)(p<0.01)$. Pacing-induced sustained ventricular tachycardia did not originate at sites of double potential formation observed during sinus rhythm that also demonstrated rate-dependent truncation and rate-dependent PVB formation with atrial pacing, but developed at other IZ epicardial sites demonstrating prolonged delays, localized block, and reentry only during the introduction of ventricular pacing at rates exceeding 300

This article is protected by copyright. All rights reserved. 
bpm.

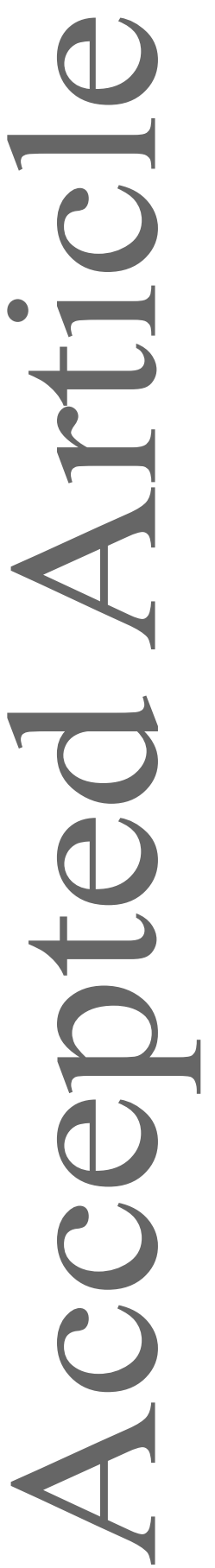

This article is protected by copyright. All rights reserved. 


\section{Discussion}

The present experiments describe constant-coupled, late-coupled ventricular PVBs observed during subacute myocardial infarction (4-5 days). The PVBs have a normal axis and a left bundle branch block ECG morphology consistent with an origin in anterior LV epicardium and early access to the His-Purkinje system. The single PVBs are associated with a splitting and decremental conduction of the terminal fragment of a fractionated or double potential IZ electrogram, following a prolonged $20-230 \mathrm{msec}$ isoelectric interval. When delayed sufficiently into late-diastole, the terminal portion of the split electrogram initiates a late-coupled PVB. Unipolar electrode mapping demonstrated earliest PVB activation across a line of apparent activation block. The apparent line of conduction block provides the substrate for slow conduction and delayed activation associated with formation of a double potential formation or a fractionated potential (Figure 6). The delayed activation across a line of apparent conduction block requires retrograde electronic activation of previously excited epicardium proximal to the arc. The extremely delayed retrograde activation is permitted by the very slow retrograde conduction through the arc of apparent antegrade conduction block, to produce the prolonged delay necessary and the prolonged isoelectric interval characteristic for late-coupled PVB formation. Although the proposed mechanism is fundamentally reentrant in nature, the conduction velocity within crucial antegrade and retrograde segments of the "circuit" is sufficiently slow so that PVB activation may be interrupted by the next sinus beat, producing PVBs that are fusion beats.

The observation of rate-dependent, late-coupled PVBs in the infarcted canine heart has been described previously. Using composite electrograms recorded 3-7 days following myocardial infarction in the dog, El-Sherif et $\mathrm{al}^{7}$ recorded examples of concealed extrasystolic grouping giving rise to ventricular extrasystoles with either fixed or variable

This article is protected by copyright. All rights reserved. 
coupling intervals. Many of the properties of arrhythmia formation demonstrated in the present experiments (1) resumption of $1: 1 \mathrm{IZ}$ delayed activation with slowing of the heart rate with vagus nerve stimulation, (2) concealed and manifest PVB formation, (3) rate-dependent progression of PVB formation from bigeminy to trigeminy to quadrigeminy with metastable PVB formation at intermediate heart rates, (4) stable Wenckebach-like patterns of IZ conduction delay associated with stable patterns of PVB formation, and (5) suppression of PVB formation at fast and slow ventricular heart rates were presented in limited examples by El-Sherif and colleagues using composite electrode recordings from ischemically-injured epicardium. El Sherif and colleagues ${ }^{7}$ proposed that the mechanism for the observation of manifest and concealed extrasystolic grouping was localized reentry with a consistent exit site for the reentrant pathway. Different anatomic reentrant pathways (despite a consistent inability to record mid-pathway electrical activity in many examples) (concealed reentry) were hypothesized to explain the different duration isoelectric periods observed with Wenckebach-like conduction delays ${ }^{8}$.

Electrophysiologic Basis for the Isoelectric Period - The basis for the very slow conduction proposed but not actually recorded during the isoelectric interval is uncertain. The mean conduction velocities and conduction delays observed with unipolar electrode mapping $(0.025 \pm 0.010 \mathrm{M} / \mathrm{sec}$, and $109 \pm 70 \mathrm{msec})$ have been in the range reported by Suenson ${ }^{9}$ for ephaptic conduction between ferret ventricular papillary muscles studied in vitro, decremental conduction through the compact $\mathrm{AV}$ node ${ }^{9}$, and reflection identified in isolated papillary muscles studied in vitro using a $2 \mathrm{~mm}$ wide poorly excitable gap ${ }^{10}$. The mean conduction velocities are slower than the mean epicardial conduction velocities measured longitudinally $(0.42 \pm 0.11 \mathrm{M} / \mathrm{sec})$ and transverse $(0.25 \pm 0.035 \mathrm{M} / \mathrm{sec})$ to epicardial muscle orientation and calculated for IZ epicardium calculated during sustained

This article is protected by copyright. All rights reserved. 
ventricular tachycardia ${ }^{11-13}$. It must however be noted that the very slow conduction velocities $<0.12 \mathrm{~mm} / \mathrm{msec}$ measured in previous in vivo studies ${ }^{11-13}$, and associated with arcs of conduction block were pre-emptively determined to represent conduction block by the investigators and were treated as such for the purpose of arrhythmia pathway mapping ${ }^{12.13}$. It is possible that very slow conduction continued through the arc of block, with early activation distal to the block pre-empted by reentrant activity arriving circuitously around the arc of block before very slow conduction arrived through the "arc of conduction block".

Prominent IZ epicardial activation delays and localized IZ conduction block at atrial heart rates $\leq 150 \mathrm{bpm}$ were associated with rate-dependent PVB formation, with arrhythmia consisting of a single PVB, never a ventricular couplet or ventricular tachycardia. Rapid, sustained ventricular tachycardia has been observed commonly in the same experimental canine model, $3-7$ days post myocardial infarction ${ }^{1,2,7,11-13}$, but is associated with sites demonstrating less prominent maximal IZ conduction delays during sinus rhythm and atrial pacing $^{14,15}$. Activation delays and conduction block sufficient to elicit sustained ventricular tachycardia at other epicardial recording sites is only observed during ventricular pacing or ventricular premature beats introduced at more rapid heart rates $(330-420 \mathrm{bpm})$, producing a reentrant pathway demonstrating slow $(<0.1 \mathrm{M} / \mathrm{sec})$ but not extremely slow conduction velocities $(<0.025 \mathrm{M} / \mathrm{sec})$. For single, late coupled PVBs, conduction block and localized reentry were formed at different anatomic sites within IZ epicardium, with extremely slow conduction evidenced by an isoelectric interval, supporting only a single "reentrant ventricular beat" ${ }^{\text {14,15 }}$.

Size of the Arrhythmia Circuit - Overall, the physical size of the isoelectric portion of the paths giving rise to late-coupled, rate-dependent PVB formation remains small, $2.4 \pm$ This article is protected by copyright. All rights reserved. 
$1.2 \mathrm{~mm}$ in the antegrade direction. The small size of the tissue pathway involved in arrhythmia generation and the prolonged isoelectric period present require an extremely slow conduction velocity. Such a slow conduction velocity would be consistent with decremental conduction within the anterior atrial transitional tissue approach to the AV node and the compact atrioventricular node ${ }^{9,16}$, activation transverse to myocardial fiber orientation during zig-zag patterns of activation through surviving strands of myocardium separated by fibrosis ${ }^{17}$, conduction across a poorly excitable or inexcitable gap within in vitro myocardial tissue ${ }^{10}$ and Purkinje fibers ${ }^{18-22}$, and ephaptic conduction between closely approximated ferret papillary muscles contained within an inexcitable gap ${ }^{8}$. In all instances when there was a $2 \mathrm{~mm}$ or greater separation of the latest site of activation for the sinus heart beat and the earliest site of PVB activation, an interposed unipolar electrode recording site failed to demonstrate local activation during the isoelectric interval.

Delay in in vitro models of reflection require either a sucrose ${ }^{18-20}$ or a hyperkalemic ${ }^{10,}$ ${ }^{18-20,23,24}$ gap. The sucrose gap (with added calcium chloride to prevent uncoupling) provides a pure electrotonic gap while hyperkalemia provides depolarization, producing both electrotonus and L-type calcium mediated "slow calcium" current ${ }^{10,18-20}$ as mechanisms for slow conduction at velocities less than $0.050 \mathrm{M} / \mathrm{sec}$. The exact basis for the slow conduction and large conduction delays over a 1-4 mm gap of epicardium in the present studies remains to be determined.

Variable Patterns of Conduction Block - The late-coupled PVBs demonstrated a characteristic pattern of stability, going from normal rhythm to bigeminy to trigeminy to quadrigeminy, with an increase in heart rate (Figures 1 and 2). At heart rates intermediate to those producing a stable pattern of PVB formation were heart rates producing metastable patterns of arrhythmia formation (Figure 1). This arrhythmia pattern is very characteristic for

This article is protected by copyright. All rights reserved. 
reflection, as produced by the interposition of an inexcitable gap in a normal papillary muscle $^{10}$ or Purkinje fiber ${ }^{18-22}$. This ex vivo arrhythmia model very closely reproduces many of the salient properties of late-coupled PVB formation in the infarcted canine heart including the characteristic pattern of rate-dependent arrhythmia formation, close apposition (1-2 $\mathrm{mm})$ of relatively normal action potential generation/conduction, and a short segment of inexcitable tissue. Although electrotonic conduction during the isoelectric interval can be provided by an inexcitable gap and may be responsible for much of the isoelectric interval, depressed sodium channels ${ }^{23}$ within the transition zones between normal and inexcitable tissues (or in the case of the L-type channels within the poorly excitable central region ${ }^{19,24}$ ) may provide the basis for some of the delay as well as the Wenckebach-like periodicity needed for arrhythmia formation. Note the fractionated electrograms subsequent to the end of surface ECG activation for the sinus beat preceding the PVB as well as preceding surface ECG activation for the PVB, at both ends of the isoelectric period.

Clinical Implications - Concealed reentry is a term developed by Langendorf and colleagues $^{25,26}$ utilizing deductive reasoning and clinical ECG records. The clinical investigators suggested that higher orders of block on the surface ECG may reflect lower orders of arrhythmia formation at localized sites in the heart. These observations and the concept of concealed reentry as an arrhythmia mechanisms was further supported by the work of other investigators ${ }^{27}$. The value of the hypothesis is not diminished when type II reflection is considered as a reentrant mechanism and is applicable for periodic arrhythmia formation described by El-Sherif et $\mathrm{al}^{7}$ as well as the present experiments. Both studies suggest a reentrant mechanism complete with a prolonged isoelectric period representing a considerable portion of the PVB coupling interval. The close approximation of the last site of sinus beat activation with the earliest site of PVB activation, despite a prolonged coupling

This article is protected by copyright. All rights reserved. 
interval would be consistent with Type II reflection as an arrhythmia mechanism. The prolonged isoelectric interval represents that significant chronological component of the coupling interval consistent with electrotonus and very slow impulse transmission.

The present rate-dependent, late-coupled single PVBs with a normal axis and left bundle branch block morphology described in the present studies may represent an interesting, yet potentially benign ventricular arrhythmia. The very slow conduction observed in the present studies is incapable of supporting the rapid sustained ventricular arrhythmias characteristic of risk for sudden coronary death. Rapid sustained arrhythmia requires a different anatomic and electrophysiologic substrate. The present data, however, provides strong evidence for type II reflection/reentry as a mechanism capable of producing ratedependent, periodic arrhythmia in the setting of healing myocardial infarction, days 4-5 following myocardial infarction in the dog.

This article is protected by copyright. All rights reserved. 


\section{References}

1. Patterson E, Scherlag BJ, Lazzara R. Arrhythmias in the canine heart, two to twenty-four hours after myocardial infarction. In Dangman KH, Miura DS, eds:

Electrophysiology and Pharmacology of the Heart. eds. New York: Marcel Dekker, 1991, pg. 301-330.

2. Mehra R, Zeiler RH, Gough WB, El Sherif N. Reentrant arrhythmias in the late myocardial infarction period. 9. Electrophysiologic-anatomic correlation of reentrant circuits. Circulation 1983;61:11-24.

3. Dillon SM, Allessie MA, Ursell PC, Wit AL. Influences of anisotropic tissue structure on reentrant circuits in the epicardial border zone of subacute canine infarction. Circ Res 1988;63:182-196.

4. Harris AS: Delayed development of ventricular ectopic rhythms following experimental coronary occlusion. Circulation 1950;1:1318-1328.

5. El-Sherif N, Gough WB, Zeiler RH, Mehra R. Triggered ventricular rhythm in 1day-old myocardial infarction in the dog. Circ Res 1983;52:566-579.

6. Patterson E, Scherlag BJ, Lazzara R. Mechanism of prevention of sudden death by nadolol; Differential actions upon arrhythmia triggers and substrate after myocardial infarction. J Am Col Cardiol 1986;8:1365-1372.

7. El-Sherif N, Lazzara R, Hope RR, Scherlag BJ. Reentrant ventricular arrhythmia in the late myocardial infarction period. 3. Manifest and concealed extrasystolic grouping. Circulation 1977; 56:225-234.

This article is protected by copyright. All rights reserved. 
8. Suenson M. Ephaptic impulse transmission between ventricular myocardial cells in vitro. Acta Physiol Scand 1984;120-445-455.

9. Hoffman BF, Cranefield PF. Electrophysiology of the Heart. Mt. Kisco, NY: Futura Publishing Co, 1960, pg. 144-143.

10. Rozanski GJ, Jalife J, Moe GK. Reflected reentry in nonhomogeneous ventricular muscle as a mechanism of cardiac arrhythmias. Circulation 1984;69:163-173.

11. Cardinal R, Vermeulen M, Shenasa M, Roberge F, Page P, Hélie F, Savard P. Anisotropic conduction and functional dissociation of ischemic tissue during reentrant ventricular tachycardia in canine myocardial infarction. Circulation. 1988;77:1162-76.

12. Ciaccio EJ, Ashikaga H, Kaba RA, Cervantes D, Hopenfeld B, Wit AL, Peters NS, McVeigh ER, Garan H, Coromilas J. Model of reentrant ventricular tachycardia based on infarct border zone geometry predicts reentrant circuit features as determined by activation mapping. Heart Rhythm 2007 4:1034-45.

13. Coromilas J, Saltman AE, Waldecker B, Dillon SM, Wit AL. Electrophysiological effects of flecainide on anisotropic conduction and reentry in infarcted canine hearts. Circulation 1995;91:2245-63.

14. Patterson E, Scherlag BJ, Berbari EJ. Rate-related ventricular ectopic beat formation in canine epicardium, four days following myocardial infarction - discordance with sustained ventricular tachycardia. PACE 1994;17:793A.

15. Patterson E, Scherlag BJ, Lander P, Berbari EJ. Double potentials following myocardial infarction: predictive value for sustained ventricular tachycardia. PACE 1996;19:708A.

This article is protected by copyright. All rights reserved. 
16. Patterson E, Scherlag BJ. Decremental conduction in the posterior and anterior AV nodal inputs. J Interv Card Electrophysiol. 2002;7:137-48.

17. de Bakker JM, van Cappelle FJ, Janse MJ, Tasseron S, Vermoulen JT, de Jonge $\mathrm{N}$, Lahpor JR. Slow conduction in the infarcted human heart: "zig-zag" course of activation. Circulation 1993;88:915-926.

18. Antzelevitch C, Jalife J, Moe GK. Characteristics of reflection as a mechanism of reentrant arrhythmias and its relationship to parasystole. Circulation 1980;61:182-191.

19. Antzelevitch C, Moe GK. Electrotonically mediated delayed conduction and reentry in relation to "slow responses" in mammalian ventricular conducting tissue. Circ Res. 1981;49:1129-39.

20. Antzelevitch C, Bernstein MJ, Feldman HN, Moe GK. Parasystole, reentry, and tachycardia: a canine preparation of cardiac arrhythmia occurring across inexcitable segments of tissue. Circulation 1983;68:1101-1115.

21. Wit AL, Hoffman BF, Cranefield PF. Slow conduction and reentry with the ventricular conduction system: I. Return Extrasystole in Cardiac Purkinje Fibers. Circ Res $1972 ; 30: 1-10$

22. Cranefield PF, Wit AL, Hoffman BF. Conduction of the cardiac impulse. III Characteristics of a very slow conduction. J Gen Physiol 1972;59:117-146.

23. Lamanna $\mathrm{V}$, Antzelevitch $\mathrm{C}$, Moe GK. Effects of lidocaine on conduction through depolarized canine false tendons and on a model of reflected reentry. J Pharmacol Exp Ther 1982;221:353-61.

This article is protected by copyright. All rights reserved. 
24. Davidenko JM, Antzelevitch C. The effects of milrinone on conduction, reflection, and automaticity in canine Purkinje fibers. Circulation 1984;69:1026-1045.

25. Mack I, Langendorf R. Factors influencing the time of appearance of premature systoles (including a demonstration of cases with ventricular premature systoles due to reentry but exhibiting variable coupling. Circulation 1950;1:910-921.

26. Langendorf R, Pick A, Winterwitz M. Mechanisms of intermittent ventricular bigeminy: I. appearance of ectopic beats dependent upon length of the ventricular cycle, the "rule of bigeminy". Circulation 1955;11:422-430.

27. Kerin N, Mori I, Levy MN. Ventricular quadrigeminy as a manifestation of concealed bigeminy. Circulation 1975;52:1023-1029.

This article is protected by copyright. All rights reserved. 
Table 1 - Unipolar Epicardial Mapping of Double Potential Formation and Rate-Dependent Arrhythmia Formation

\begin{tabular}{|l|c|c|}
\hline $\begin{array}{l}\text { Distance from Site of Latest } \\
\text { Activation }(\mathrm{mm})\end{array}$ & $\begin{array}{c}\text { Mean Conduction Velocity Into } \\
\text { PVB Origin }(\mathrm{mm} / \mathrm{msec})\end{array}$ & $\begin{array}{c}\text { Mean Conduction Velocity } \\
\text { Out of PVB Origin }(\mathrm{mm} / \mathrm{sec})\end{array}$ \\
\hline $1.0 \mathrm{~mm}(\mathrm{~N}=2)$ & $0.050,0.014$ & $0.025,0.017$ \\
\hline $1.4 \mathrm{~mm}(\mathrm{~N}=2)$ & $0.050,0.026$ & $0.045,0.045$ \\
\hline $2.0 \mathrm{~mm}(\mathrm{~N}=1)$ & 0.020 & 0.050 \\
\hline $2.8 \mathrm{~mm}(\mathrm{~N}=3)$ & $0.070,0.025,0.017$ & $0.050,0.045$ \\
\hline $4.0 \mathrm{~mm}(\mathrm{~N}=2)$ & $0.050,0.023$ & $0.050,0.035$ \\
\hline & & $0.045 \pm 0.020$ \\
\hline $\mathrm{X} \pm \mathrm{SD}=2.3 \pm 1.1$ & $0.025 \pm 0.010$ & \\
\hline
\end{tabular}

This article is protected by copyright. All rights reserved. 
Figure 2

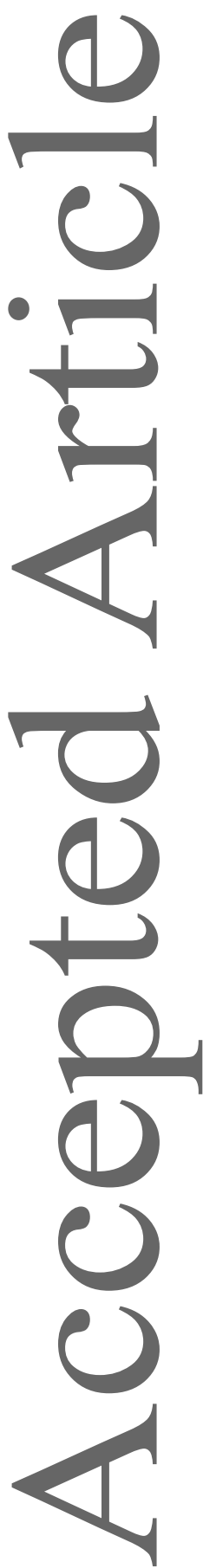

This article is protected by copyright. All rights reserved. 
Figure 3

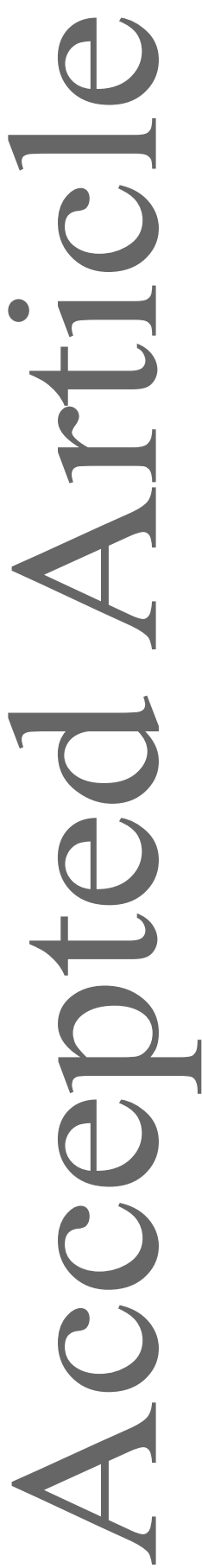

This article is protected by copyright. All rights reserved. 
A. Sinus Rhythm

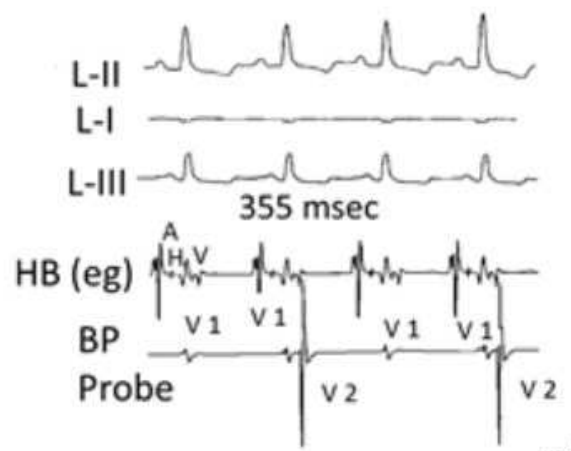

B. Vagus Nerve Stimulation

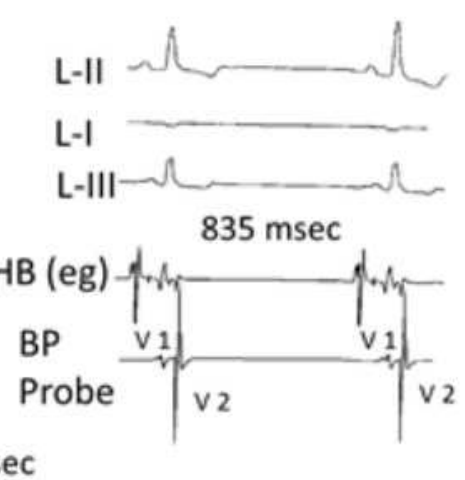

$20 \overline{\mathrm{msec}}$

C. Vagus Nerve Stimulation PVB

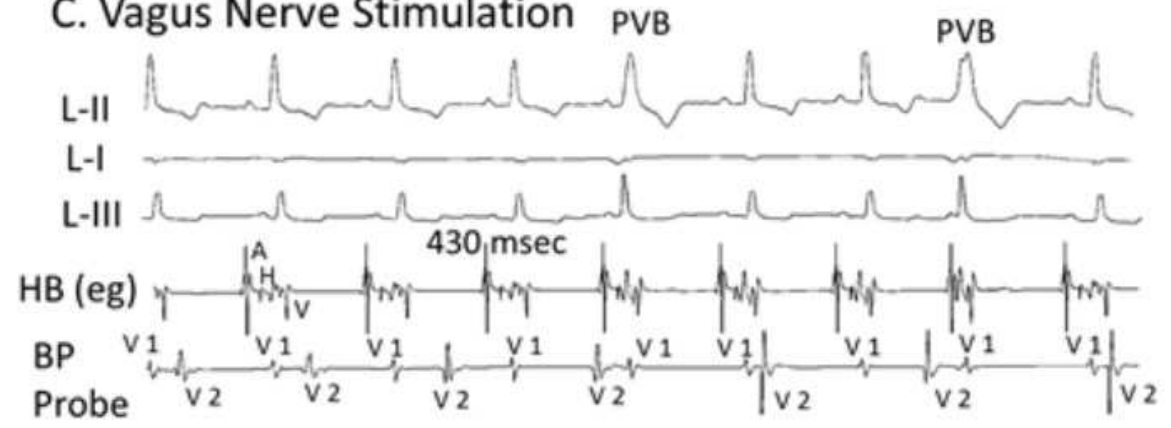

D.

E. Sinus Rhythm Atrial-Paced Beat PVC PVC PACING

I
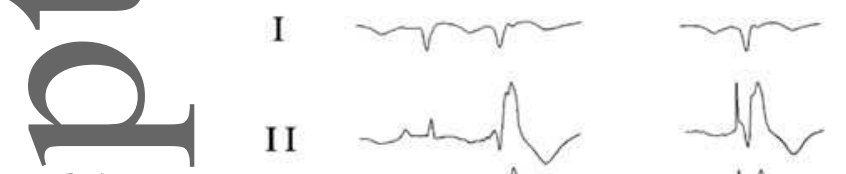

II

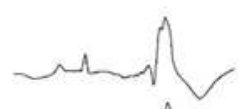

III

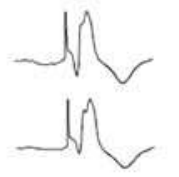

8

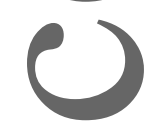

A V R

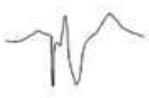

A V L

A VF

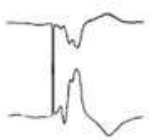

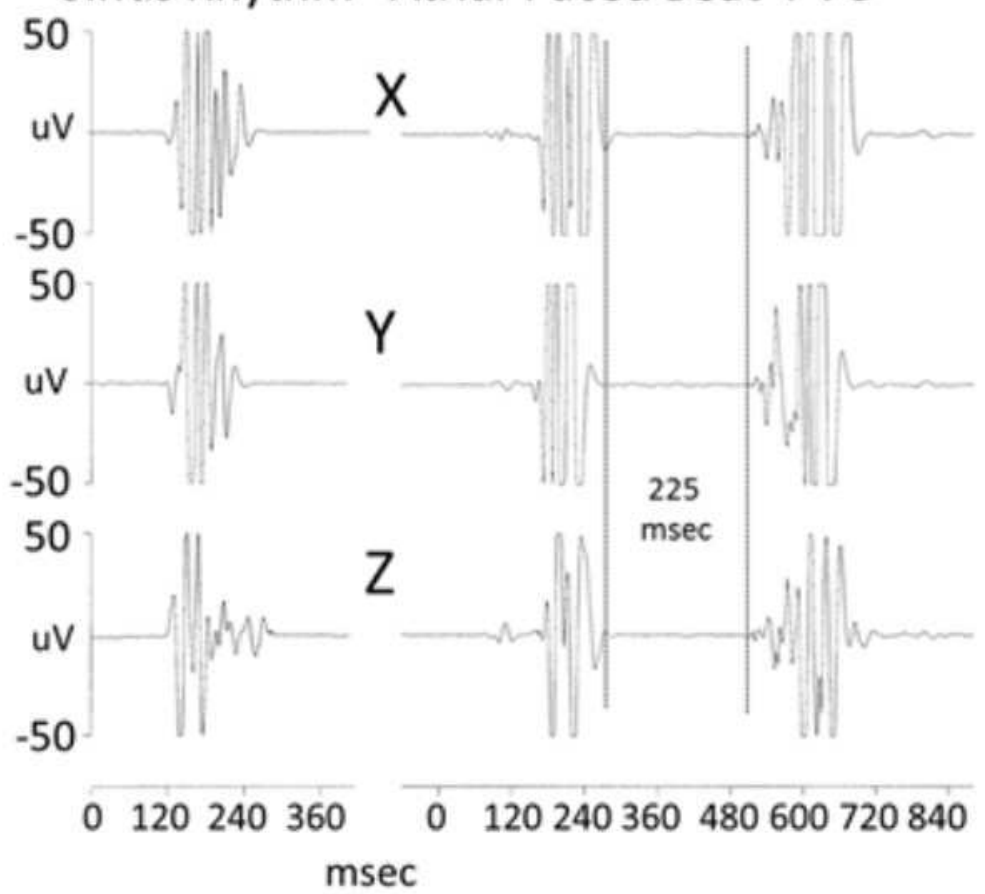

This article is protected by copyright. All rights reserved. 
Figure 5

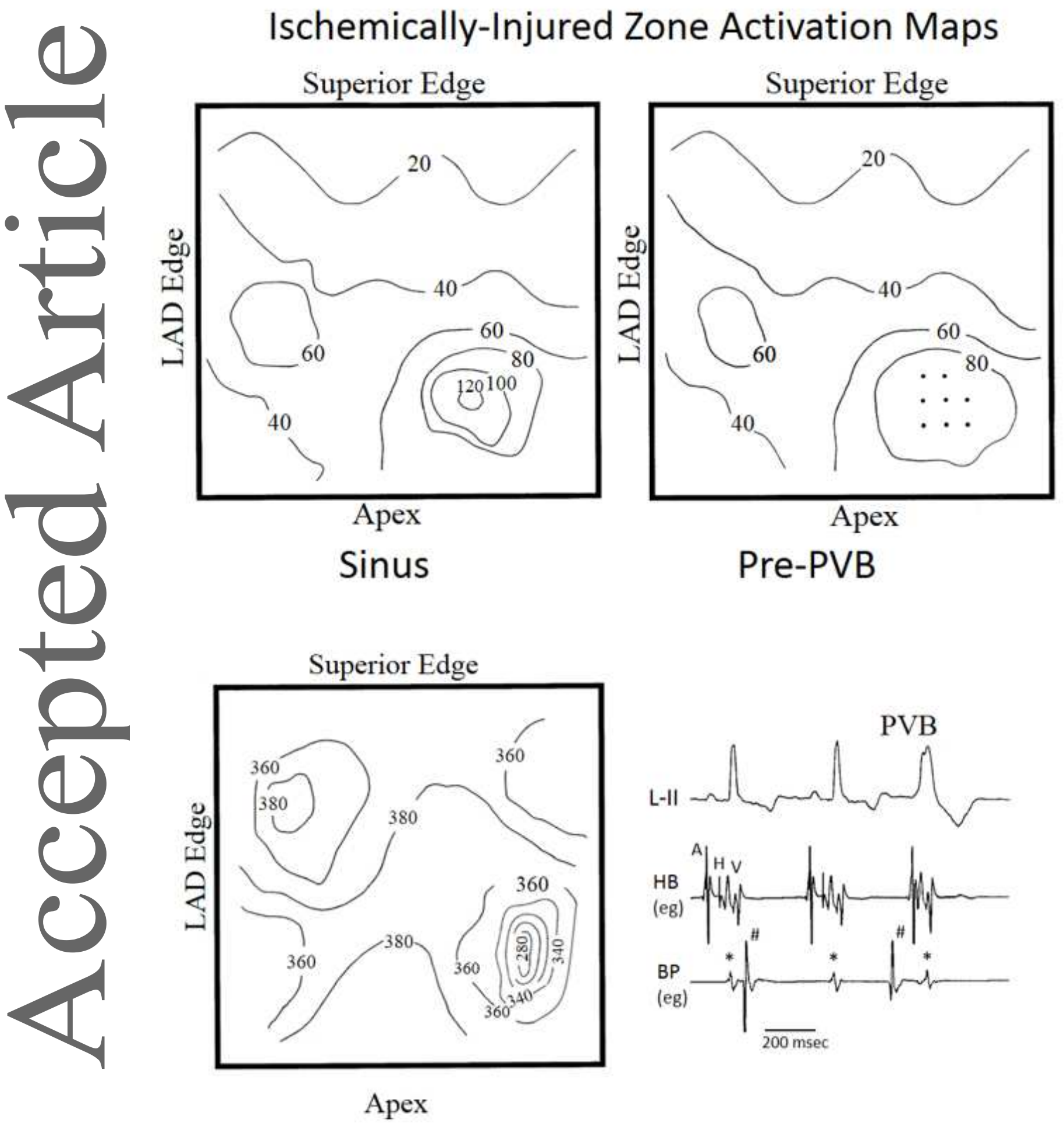

This article is protected by copyright. All rights reserved. 
Figure 6

\section{Antegrade Conduction Through Apparent Arc of Conduction Block}

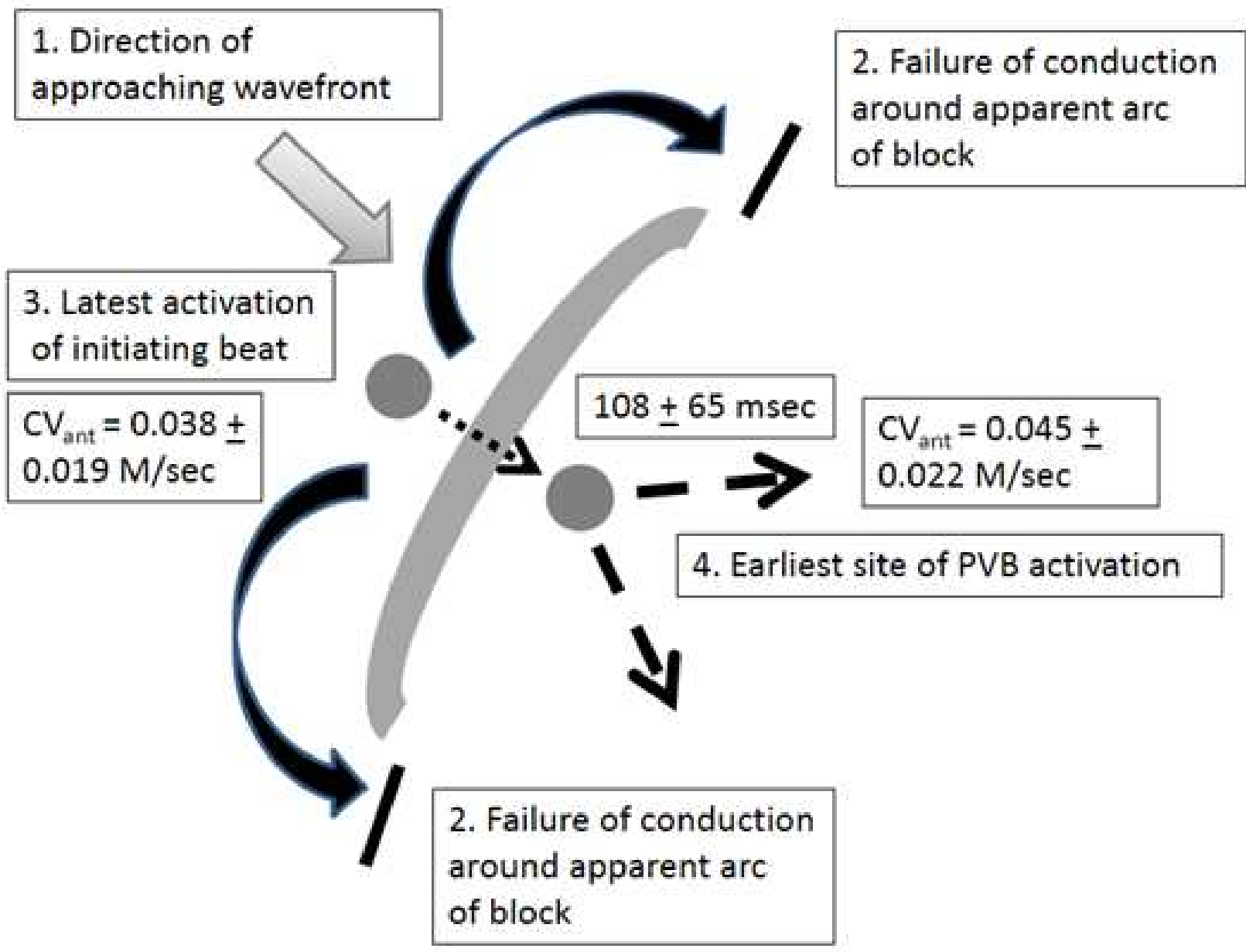

Figure 1 - Patterns of Rate-dependent Ventricular Ectopy Formation and Periodic PVB Formation as a Function of Ventricular Cycle Length - In Figure 1A, individual patterns of 2:1, 3:1, and 4:1 PVB formation are shown in a lead II ECG during sinus rhythm observed at spontaneous cycle lengths of 722,600 , and $570 \mathrm{msec}$, respectively, during a single

This article is protected by copyright. All rights reserved. 
experiment. The PVB is late-coupled (550 msec) at a sinus cycle length of $570 \mathrm{msec}$, producing a fusion beat with the subsequent sinus heartbeat. In Figure 1B, PVB formation as a percentage of the total ventricular beats is shown as a function of the ventricular cycle length for a single representative experiment. Stable patterns of $2: 1,3: 1$, and $4: 1$ block as shown above in panel A were observed with increases in the ventricular rate during atrial pacing.

Figure 2 - Periodic PVB Formation as a Function of Ventricular Cycle Length - Figure 2 (panels A-D shows electrophysiologic recordings from the anesthetized dog, 4 days postcoronary artery occlusion during vagus nerve stimulation (panel A), right atrial pacing at a 425 msec cycle length (panel C), and sinus rhythm (panel D). A lead II ECG (L-2), His bundle electrogram (HB(eg)), normal zone (NZ) and ischemically-injured zone (IZ) composite electrograms, $\mathrm{X}, \mathrm{Y}$, and $\mathrm{Z}$ bipolar orthogonal electrograms from the borders of the infarct zone, and an endocardial bipolar electrogram from the $I Z(P)(e g)$ are shown during vagus nerve stimulation (atrial cycle length $=760 \mathrm{msec})($ panel A). No periodic conduction of delayed IZ activity and no ventricular ectopic beats are present. An enlarged view of the IZ composite electrogram and $\mathrm{X}$ orthogonal lead bipolar electrogram (dashed box) are shown in the upper part of panel B (vagus nerve stimulation). In panel C, at a paced right atrial cycle length of $425 \mathrm{msec}$, the late portions of the portions of the IZ composite and X orthogonal electrograms fractionate and delay (marked by asterisks) producing 2:1 periodic and delayed activation with late-coupled PVB formation (3:1). An enlargement of the IZ composite and $\mathrm{X}$ bipolar orthogonal electrogram recordings (dashed box) is shown in the bottom half of panel B. The same potential during sinus rhythm (488 msec cycle length) (panel D) demonstrate a 3:2 pattern of progressive delays (best observed in $\mathrm{X}$ orthogonal electrode recording) producing 3:1 pattern of PVB formation. The prolonged, fractionated,

This article is protected by copyright. All rights reserved. 
and delayed electrical activity best seen in the $\mathrm{X}$ orthogonal electrogram is delayed moderately, failing to exceed the T wave on the second beat and failing to initiate a PVB (panel D). The delay is greater for the third beat, exceeding the QT segment, and initiating a late coupled PVB (panel D).

Figure 3 - Bipolar Electrode Recordings From Ischemically-Injured (IZ) Epicardium, 4 Days Post Coronary Artery Occlusion (panels A-C), Pace Mapping (panel D), and Signal Averaging of Local Bipolar Orthogonal Leads Recorded From the Infarct Borders (panel E) - Lead I, II, and III ECGs (L-1, L-II, and L-III), His-bundle electrogram $(\mathrm{HB}(\mathrm{eg}))$, and bipolar electrode recordings of IZ epicardium from a hand-held probe (BP Probe) are shown for sinus rhythm (panel A)(sinus cycle length $=355 \mathrm{msec}$ ), during vagus nerve stimulation (panel $B$ )(sinus cycle length $=835 \mathrm{msec}$ ), and during vagus nerve stimulation (panel C)(sinus cycle length $=430 \mathrm{msec}$ ). Stable $2: 1$ conduction block of the $2^{\text {nd }}$ potential of the double potential is observed in panel $A$, without arrhythmia formation. $1: 1$ Conduction of the $2^{\text {nd }}$ potential of the double potential in panel $B$ at a more prolonged ( 835 $\mathrm{msec}$ ) cycle length also fails to produce PVB formation. Periodic delay of the $2^{\text {nd }}$ potential of the double potentials, beyond the T wave of the surface ECG, produces PVB formation as fusion beats (panel C) (PVBs). In Panel D is shown a six-lead ECG of a spontaneous PVB reproduced in a repetitive manner. A bipolar electrode was used to record a double potential from IZ epicardium, with splitting of the double potential giving rise to the PVB (left hand panel). Also shown are the same 6 ECG leads recorded during bipolar pacing from the site of double potential formation on the epicardial surface overlying myocardial infarction, reproducing the same ECG pattern (right hand panel) as seen with spontaneous PVBs. Panel $E$ shows $X, Y$, and $Z$ leads from orthogonal lead recorded from the borders of the anterior infarct. During sinus rhythm (left hand side of panel E), a signal-averaged beat from

This article is protected by copyright. All rights reserved. 
the $\mathrm{IZ}$ zone is shown for $\mathrm{X}, \mathrm{Y}$, and $\mathrm{Z}$ orthogonal electrodes showing significant low-voltage delayed activation most evident in the $Z$ electrogram. In the right-hand side of panel $E$, a signal-averaged atrially-paced beat and subsequent constant-coupled PVB are shown, with a 225 msec duration isoelectric period between the atrially-paced beat and the PVB. The recording was the average of 200 PVBs.

\section{Figure 4 - Activation Maps for a $12 \times 12 \mathrm{~mm}$ Area of Ventricular Epicardium Overlying} Ischemically-Injured Ventricular Epicardium, 4 Days Post Coronary Artery Occlusion During Sinus Rhythm, During Vagus Nerve Stimulation, During the Beat Preceding the Premature Ventricular Beat (Pre PVB), and During the Premature Ventricular Beat (PVB) - Isochronal unipolar activation maps for sinus rhythm (Sinus)(large upper left-hand panel) and during vagus nerve stimulation (Vagus Nerve Stimulation)(large upper right-hand panel) are shown for an $11 \times 11$ array having a $1 \mathrm{~mm}$ electrode separation. Bipolar electrode recordings taken from IZ epicardium at the site of arrhythmia formation are also shown (small panels). During sinus rhythm a maximal delay of $124 \mathrm{msec}$ was recorded and during vagus nerve stimulation a maximal delay of $102 \mathrm{msec}$ was recorded. A double potential was present in both closely-spaced bipolar electrode recordings. Isochronal unipolar activation maps are also shown during atrial pacing (385 msec cycle length) that produced ventricular trigeminy (lower panels). The ventricular beat of atrial origin immediately preceding the PVB is shown in the large lower left hand panel with the latest site of activation being 126 msec. The earliest site of activation for the PVB occurred across an apparent line of conduction block (closely spaced isochrones), only $2 \mathrm{~mm}$ from the site of latest activation for the previous beat, but separated by an isoelectric interval lasting $94 \mathrm{msec}$ (large lower right hand panel).

This article is protected by copyright. All rights reserved. 
Figure 5 - Activation Maps for a $12 \times 12 \mathrm{~mm}$ Area of Ventricular Epicardium Overlying Ischemically-Injured Ventricular Epicardium, 4 Days Post Coronary Artery Occlusion for Sinus Rhythm, the Beat Preceding the Premature Ventricular Beat (Pre-PVB), and the Premature Ventricular Beat - Unipolar isochronal activation maps are shown during sinus rhythm for beat one (Sinus)(upper left hand panel) and beat 2 immediately preceding the PVB (pre-PVB)(upper right hand panel) of ventricular trigeminy, and during the PVB (lower left panel). The latest site of ventricular activation (128 msec) (upper left-hand panel) and seven other unipolar recording sites were not activated during beat 2 (upper right-hand panel) until following an isoelectric period lasting $190 \mathrm{msec}$. A distance of $1 \mathrm{~mm}$ separates the sites of latest activity of the pre-initiating beat and the earliest recorded electrical activity of the PVB. The right- hand panel shows a lead II ECG (L-II), His bundle electrogram $(\mathrm{HB}(\mathrm{eg}))$, and bipolar electrogram from IZ epicardium $(\mathrm{BP}(\mathrm{eg}))$ during ventricular trigeminy recorded previously during the experiment showing splitting and delayed conduction of the bipolar electrogram associated with PVB formation.

\section{Figure 6 - Antegrade Conduction Through Apparent Arc of Conduction Block as an}

Arrhythmia Mechanism - An approaching wave front produces an arc of conduction block and forces the wave front to conduct around an arc of conduction block. For the 10 examples studied using a dense $11 \times 11$ unipolar electrode array), conduction around the arc of block is so delayed or is completely blocked (2) that very slow conduction through the arc of apparent conduction block, taking $108 \pm 65 \mathrm{msec}$ to travel $2.4 \pm 1.2 \mathrm{~mm}$ at $0.038 \pm$ $0.020 \mathrm{M} / \mathrm{sec}$, arrives before any conduction around the arc of apparent conduction block. Initial conduction for the first 1-2 mm from the earliest site of PVB activation (4) is also very slow $(0.045 \pm 0.022 \mathrm{M} / \mathrm{sec})$.

This article is protected by copyright. All rights reserved. 